\title{
Energy and centrality dependences of charged multiplicity density in relativistic nuclear collisions
}

\author{
Ben-Hao Sa ${ }^{1,5,6 *}$ A. Bonasera ${ }^{2 \dagger}, \mathrm{An} \mathrm{Tai}^{3}$, and Dai-Mei Zhou ${ }^{4}$ \\ 1 China Institute of Atomic Energy, \\ P. O. Box 275 (18), Beijing, 102413 China \\ ${ }^{2}$ Laboratorio Nazionale del Sud, Instituto Nazionale Di Fisica Nucleare, \\ v. S. Sofia 4495132 Catania, Italy \\ ${ }^{3}$ Department of Physics and Astronomy, University of California, \\ at Los Angeles, Los Angeles, CA 90095 USA \\ ${ }^{4}$ Institute of Particle Physics, Huazhong Normal University, Wuhan, 430079 China \\ ${ }^{5}$ CCAST (World Lab.), P. O. Box 8730 Beijing, 100080 China \\ ${ }^{6}$ Institute of Theoretical Physics, Academia Sinica, Beijing, 100080 China
}

\begin{abstract}
Using a hadron and string cascade model, JPCIAE, the energy and centrality dependences of charged particle pseudorapidity density in relativistic nuclear collisions were studied. Within the framework of this model, both the relativistic $p+\bar{p}$ experimental data and the PHOBOS and PHENIX $A u+A u$ data at $\sqrt{s}_{n n}=130 \mathrm{GeV}$ could be reproduced fairly well without retuning the model parameters. The predictions for full RHIC energy $A u+A u$ collisions and for $P b+P b$ collisions at the ALICE energy were given. Participant nucleon distributions were calculated based on different methods. It was found that the number of participant nucleons, $\left\langle N_{\text {part }}\right\rangle$, is not a well defined variable both experimentally and theoretically. Therefore, it is inappropriate to use charged particle pseudorapidity density per participant pair as a function of $\left\langle N_{\text {part }}\right\rangle$ for distinguishing various theoretical models.
\end{abstract}

PACS numbers: 25.75.Dw, 24.10.Lx, 24.85.+p

\footnotetext{
*E-mail: sabh@iris.ciae.ac.cn

$\dagger$ E-mail: bonasera@lns.infn.it
} 
The main focus of the Relativistic Heavy-Ion Collider (RHIC) at Brookhaven National Laboratory (BNL) is to explore the phase transition related to the quark deconfinement and the chiral symmetry restoration. The first available experimental data were the energy dependence of charged particle pseudorapidity density in $A u+A u$ collisions at $\sqrt{s}_{n n}=56$ and $130 \mathrm{GeV}$ from the PHOBOS collaboration [1]. After that, the PHENIX collaboration published their data of centrality dependence of the charged particle pseudorapidity density in $A u+A u$ collisions at $\sqrt{s}_{n n}=130 \mathrm{GeV}$ [2].
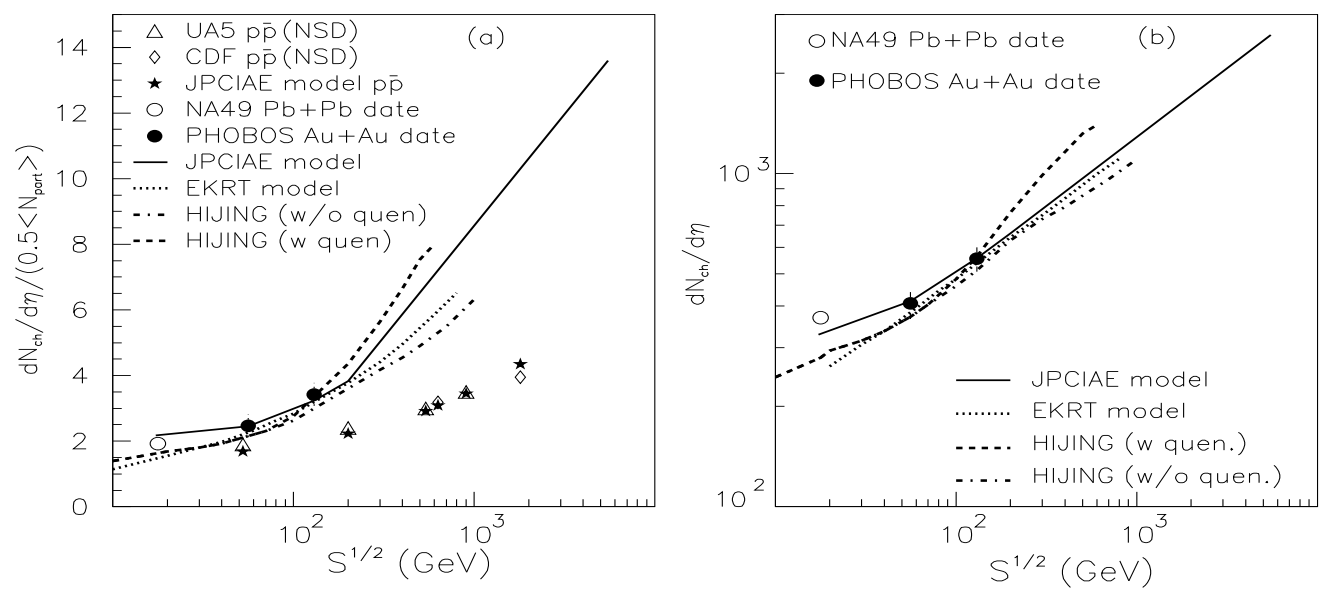

FIG. 1: The energy dependence of the charged particle pseudorapidity density at midpseudorapidity in relativistic $p+\bar{p}$ and $A+A$ collisions.

It has been predicted that the rare high charged multiplicity could indicate the onset of the Quark-Gluon-Plasma (QGP) phase, since the extra entropy in the QGP phase could

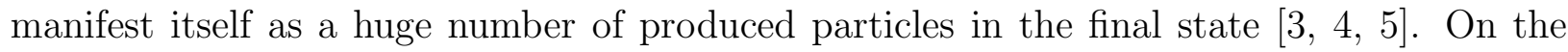
other hand, in [6] the centrality dependence of charged multiplicity has been proposed to provide information on the relative importance of soft versus hard processes in particle production and therefore provide a means of distinguishing various theoretical models for particle production.

The pQCD calculation with assumption of gluon saturation [7] (referred to as EKRT model later) was first used to study the centrality dependence of the charged particle pseudorapidity density at RHIC. In [6] the HIJING model met with success in describing both the energy and centrality dependence of the charged particle pseudorapidity density. The conventional eikonal approach and the high density QCD (referred to as KN model later) [8] were also used to investigate the centrality dependence and the both methods surpris- 
ingly obtained almost identical centrality dependence. Recently, authors in [9] reported their results from the Dual Parton Model. It was found that the experimental observation, the charged particle pseudorapidity density per participant pair slightly increasing with $<N_{\text {part }}>$, was reproduced by [6, 8, 9], but contradicted the results of [0].

In this letter a hadron and string cascade model, JPCIAE [10], was employed to study this issue further. Within the framework of this model the experimentally measured energy dependence of the charged particle mid-pseudorapidity density per participant pair both in relativistic $p+\bar{p}$ and $A u+A u$ collisions at RHIC was reproduced fairly well without retuning the model parameters. The predictions for the full RHIC energy $A u+A u$ collisions and for $\mathrm{Pb}+\mathrm{Pb}$ collisions at the ALICE energy were also given. In studying centrality dependence the focus was put on the calculations of $\left\langle N_{\text {part }}\right\rangle$, its definition and uncertainty. Both the PHENIX [2] and the PHOBOS [11] observations that the charged particle mid-pseudorapidity density per participant pair slightly increases with $\left\langle N_{\text {part }}\right\rangle$ could be reproduced fairly well by JPCIAE. However, this study indicated that it is not suitable to use the charged particle mid-pseudorapidity density per participant pair as a function of $<N_{\text {part }}>$ to constrain theoretical models for particle production, because $\left\langle N_{\text {part }}\right\rangle$ is not a well defined physical variable both experimentally and theoretically.

The JPCIAE model was developed based on PYTHIA [12]. In the JPCIAE model the nucleons in a colliding nucleus are distributed randomly in the sphere of the nucleus with a radius of $1.12 A^{1 / 3} \mathrm{fm}$. The modules of the nucleons are sampled by the Woods-Saxon distribution and the solid angles of the nucleons are sampled uniformly in $4 \pi$. Each nucleon is given a beam momentum in $\mathrm{z}$ direction and zero initial momentum in $\mathrm{x}$ and $\mathrm{y}$ directions. After the construction of initial particle list the collision time of each colliding pair is calculated under the requirement that the least approaching distance of the colliding pair along their Newton straight-line trajectory should be smaller than $\sqrt{\sigma_{t o t} / \pi}$, where $\sigma_{t o t}$ refers to the total cross section. The nucleon-nucleon collision with the least collision time is then selected from the initial collision list to perform the first collision. After the first collision, both the particle list and the collision list are updated and now the collision list may consist of not only nucleon-nucleon collisions, but also collisions between produced particles and the nucleons and between produced particles themselves. The next collision is selected from the new collision list. The processes proceed until the collision list is empty.

For each collision pair, if its CMS energy is larger than a given cut, we assume that 
strings are formed after the collision and PYTHIA is used to deal with particle production. Otherwise, the collision is treated as a two-body collision [13, 14, 15]. The cut (=4 GeV in the program) was chosen by observing that JPCIAE correctly reproduces charged multiplicity distributions in AA collisions.

It should be noted here that the JPCIAE model is not a simple superposition of nucleonnucleon collisions since the rescatterings among participant, spectator nucleons and produced particles are taken into account. We refer to [10] for more details of the JPCIAE model.
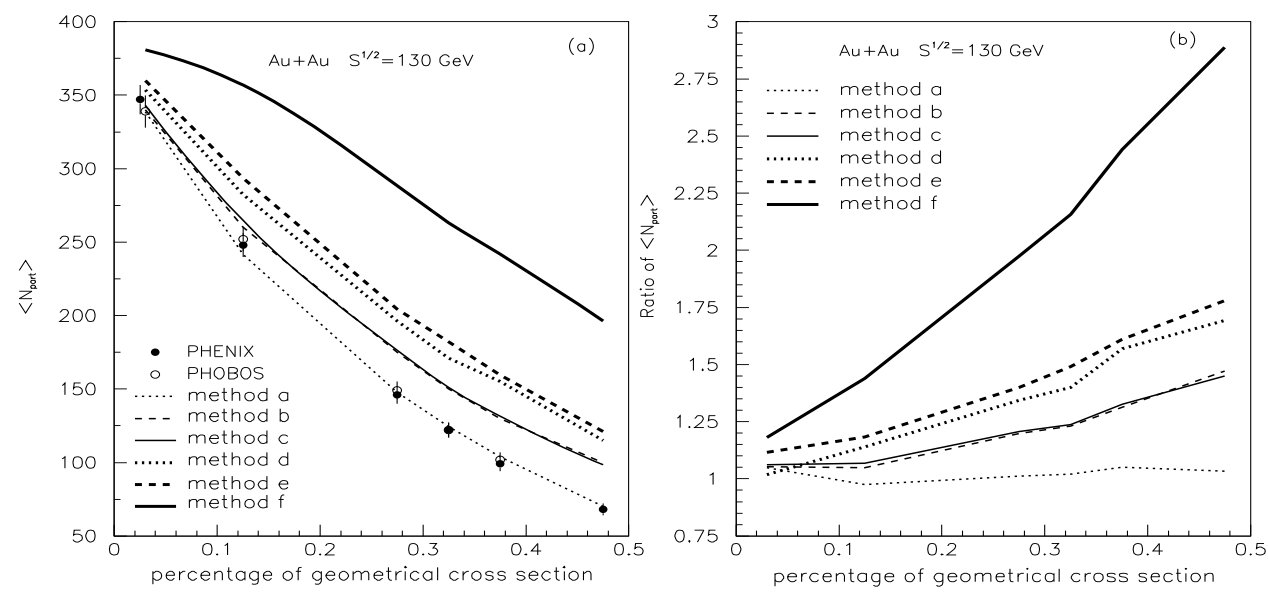

FIG. 2: The number of participant nucleons $\left\langle N_{\text {part }}\right\rangle$, (a), and the ratios of the different curves in (a) to the PHENIX data, (b), as a function of the percentage of geometrical cross section.

In Fig.1(a) the experimental data of charged particle pseudorapidity density per participant pair at mid-pseudorapidity in relativistic $p+\bar{p}$ (open triangles and rhombuses with error bar) and $A+A$ collisions (open circles with error bar for $P b+P b$ at SPS and full circles with error bar for $A u+A u$ at RHIC) [1] are compared with JPCIAE model (full stars for $p+\bar{p}$ and the solid curve for $A+A$ collisions), HIJING model (dotted-dash curve without jet quenching and dashed curve with jet quenching) [6 and EKRT model (dotted curve) [7]. The data of both $p+\bar{p}$ and $A+A$ collisions at relativistic energies were reproduced fairly well by JPCIAE model without retuning model parameters. Fig. 1 (b) is the same as (a) but the vertical coordinate here is the charged particle pseudorapidity density itself. The JPCIAE model predictions for full RHIC energy $A u+A u$ collisions and for $P b+P b$ collisions at the ALICE energy in both panels may supply a benchmark for QGP formation since the QGP phase is not included in the JPCIAE model.

Since number of participant nucleons, $\left\langle N_{\text {part }}\right\rangle$, plays a crucial role in the presentation 
of PHOBOS or PHENIX centrality dependence data we first make a study on $\left\langle N_{\text {part }}\right\rangle$. In the fixed target experiments the participant nucleons from the projectile nucleus with atomic number $A$, for instance, is estimated from

$$
N_{\text {part }}^{p}=A *\left(1-\frac{E_{Z D C}}{E_{\text {beam }}^{\text {kin }}}\right)
$$

where $E_{Z D C}$ refers to the energy deposited in the Zero Degree Calorimeter dominated by projectile spectator nucleons and $E_{\text {beam }}^{\text {kin }}$ is the kinetic energy of beam [16]. However, in the collider experiments, in order to obtain $\left\langle N_{\text {part }}\right\rangle$ one has to relate the measurables to Monte Carlo simulations. In PHENIX, for instance, simulations for the response of the Beam-Beam Counter and the ZDC were used to calculate $<N_{\text {part }}>$ via a Glauber model [2]. In PHOBOS $<N_{\text {part }}>$ is derived relating HIJING simulations to the signals in the paddle counter [1].

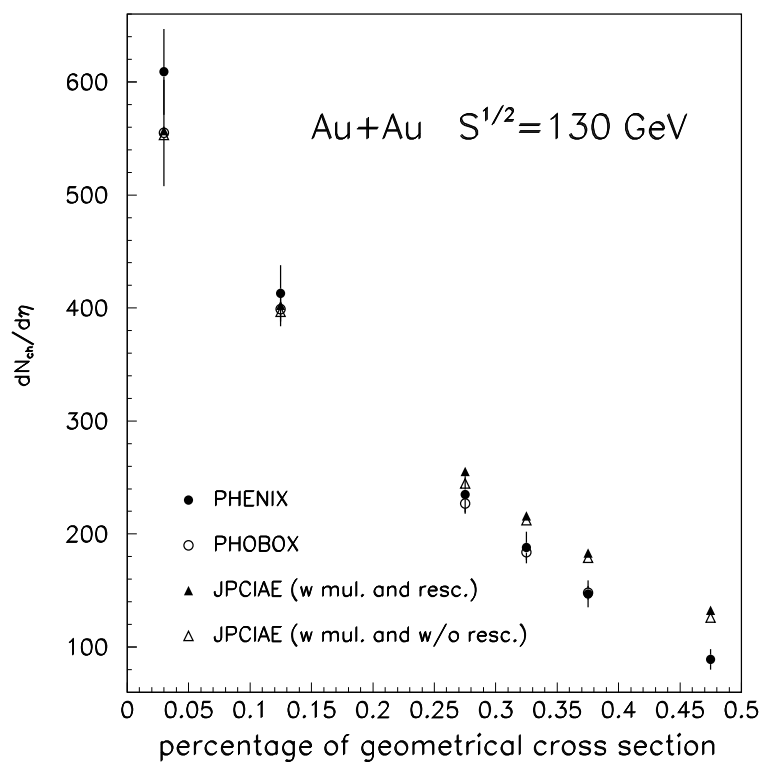

FIG. 3: The charged particle pseudorapidity density at mid-pseudorapidity in $A u+A u$ collisions at $\sqrt{s}_{n n}=130 \mathrm{GeV}$ as a function of the percentage of geometrical cross section.

On the theory side, first, $\left\langle N_{\text {part }}>\right.$ could be calculated geometrically (referred to as method a later) [17], number of participant nucleons from the projectile nucleus, for instance, reads

$$
N_{\text {part }}^{p}(b)=\rho_{0} \int d V \theta\left(R_{p}-\left[x^{2}+(b-y)^{2}+z^{2}\right]^{1 / 2}\right) \theta\left(R_{T}-\left(x^{2}+y^{2}\right)^{1 / 2}\right) .
$$

Second, in the Glauber model $N_{\text {part }}$ is calculated by (referred to as method b later) [7]

$$
N_{\text {part }}^{p}(b)=\int d^{2} s T_{A}(\vec{b}-\vec{s})\left[1-\exp \left(-\sigma_{i n} T_{B}(\vec{s})\right)\right]+\int d^{2} s T_{B}(\vec{s})\left[1-\exp \left(-\sigma_{i n} T_{A}(\vec{b}-\vec{s})\right)\right]
$$


where $\sigma_{i n} \approx 40 \mathrm{mb}$ is the inelastic nn cross section at RHIC and $T_{A}$ refers to the nuclear thickness function of nucleus A. The third method is to count the participant or the spectator nucleons in the simulation for nuclear collisions and then to average over simulated events. However, there is multifarious in simulating programs and even in the definition of the participants and spectators. FRITIOF 7.02 (referred to as method d later) [18] was popularly employed in the past. In FRITIOF the wounded nucleons, i.e., nucleons which suffer at least one inelastic collision, are counted and identified as $\left\langle N_{\text {part }}\right\rangle$. It should be pointed out here that in FRITIOF leading nucleons undergo multiple scatterings and get excited (forming strings) during collisions, but produced particles from the string fragmentation do not have rescattering. Unlike JPCIAE, FRITIOF is not a transport model, there is no space-time coordinates associated with each particle. In JPCIAE simulations we have devised three counting methods: First, the leading nucleons involved in at least one inelastic nucleonnucleon collision with string excitation are counted and identified as $\left\langle N_{\text {part }}\right\rangle$. This is called method c. It should be mentioned that the JPCIAE results in Fig. 1 were calculated by $\left\langle N_{\text {part }}\right\rangle$ from method c. Second, in method e, spectator nucleons are counted at the final state of JPCIAE simulations without rescattering (i.e., only nucleon-nucleon collisions are included), then $\left.<N_{\text {part }}\right\rangle$ is calculated through

$$
<N_{\text {part }}>=(A+B)-<N_{\text {spec }}>,
$$

where $\mathrm{A}$ and $\mathrm{B}$ refer to the atomic numbers of the target and projectile nuclei. Third, method $\mathrm{f}$ is the same as method e but JPCIAE simulations are with rescattering. The difference among method $\mathrm{c}, \mathrm{e}$, and $\mathrm{f}$ is that those nucleons which only experience two-body nucleon-nucleon collisions (without string formation) are included into $\left\langle N_{\text {part }}\right\rangle$ in method $\mathrm{e}$, while in method $\mathrm{f}$ even those nucleons which suffer collisions with other produced particles are also included into $\left\langle N_{\text {part }}\right\rangle$. In other word, in method e and $\mathrm{f}$ the nucleons that are knocked out of the colliding nuclei by the produced particles are included into $\left\langle N_{\text {part }}\right\rangle$. In emulsion chamber experiment, such nucleons (protons) are usually called 'grey tracks'.

Fig. 2 (a) gives $\left\langle N_{\text {part }}\right\rangle$ calculated by different methods as a function of percentage of the geometrical (total) cross section and compares them with results of PHENIX (solid circles with error bar) [2] and of PHOBOS (open circles with error bar) [11]. The thin dotted, dashed, and solid curves in this panel are the results of method a (geometry), b (Glauber model, taken from [7]), and c, respectively. Thick dotted, dashed, and solid 
curves are, respectively, the results of method d (FRITIOF), e, and f. One knows from Fig. 2 (a) that except method $\mathrm{f}<N_{\text {part }}>$ from different methods are close to each other (the 10\%-15\% difference should contribute to the systematic error of the experimentallyextracted $\left\langle N_{\text {part }}>\right.$ ) for most central collisions but the discrepancies among them increase with decreasing centrality in general. It is surprising that the results of geometry method are closest to the results of PHINEX or PHOBOS. In Fig. 2 (b) the ratios of $\left\langle N_{\text {part }}\right\rangle$ from methods a, b, c, d, e and f to the corresponding results of PHENIX are given.

The charged particle pseudorapidity density at mid-pseudorapidity in $A u+A u$ collisions at $\sqrt{s}_{n n}=130 \mathrm{GeV}$ as a function of the percentage of geometrical cross section is given in Fig. 3. In this figure the full and open circles with error bar are the PHENIX [2] and PHOBOS [11] data, respectively. The full and open triangles, respectively, are the results of JPCIAE model with rescattering and without rescattering. The abscissa of the data point in this figure is set at the middle of the corresponding bin of percentage of the geometrical cross section. Globally speaking, the experimental data were reproduced fairly well by the JPCIAE model. However, the agreement between the experimental data and the JPCIAE model becomes less satisfied for peripheral collisions. One knows from this figure that the rescattering only leads to a few percent increase in charged multiplicity although rescattering might enhance yields of strangeness, $\Xi^{-}+\overline{\Xi^{-}}$for instance, by a couple of times.
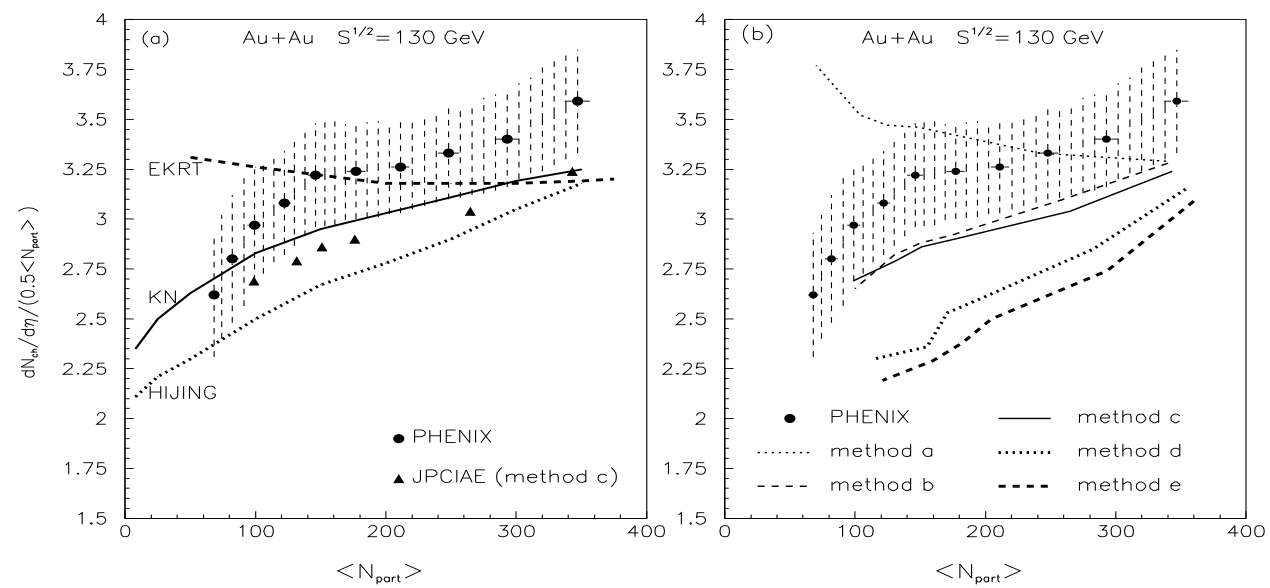

FIG. 4: The charged particle pseudorapidity density per participant pair at mid-pseudorapidity in $A u+A u$ collisions at $\sqrt{s}_{n n}=130 \mathrm{GeV}$ as a function of the number of participant nucleons, $<N_{\text {part }}>$.

In panel (a) of Fig. 4 we compare the PHENIX data of the charged particle mid- 
pseudorapidity density per participant pair (full circles with shaded area of systematic errors) [2] with JPCIAE model (full triangles, $\left\langle N_{\text {part }}\right\rangle$ from method c) and with HIJING model (dotted curve), KN model (solid curve), and EKRT model (dashed curve). One can see that except EKRT, all the other three models predict an increase of $\left(d n_{c h} /\left.d \eta\right|_{\eta=0}\right) /\left(0.5<N_{\text {part }}>\right)$ as a function of $\left\langle N_{\text {part }}>\right.$ though the theoretical results seem to underestimate the PHENIX data. Fig. 4 (b) compares the PHENIX data to the JPCIAE results of $\left\langle N_{\text {part }}\right\rangle$ calculated by method a (thin dotted curve), b (thin dashed curve), c (thin solid curve), d (thick dotted curve), and e (thick dashed curve), respectively. One sees from this panel that starting from a single result of charged particle mid-pseudorapidity density from the JPCIAE model but using $<N_{\text {part }}>$ from different methods, it is possible to lead $\left(d n_{c h} /\left.d \eta\right|_{\eta=0}\right) /\left(0.5<N_{\text {part }}>\right)$ to either increase or decrease with the increase of $\left\langle N_{\text {part }}\right\rangle$. Although $\left\langle N_{\text {part }}\right\rangle$ from method a are closest to the PHENIX results (cf. Fig. 2 (b)) the $\left(d n_{c h} /\left.d \eta\right|_{\eta=0}\right) /\left(0.5<N_{\text {part }}>\right.$ ) from JPCIAE have actually centrality dependence opposite to the PHENIX result (cf. Fig.4 (b)) because the $\left(d n_{c h} /\left.d \eta\right|_{\eta=0}\right)$ from JPCIAE is higher than the PHENIX result for peripheral collisions (cf. Fig. 3). On the other hand, even though the discrepancy between $\left\langle N_{\text {part }}\right\rangle$ from method c and PHENIX slightly increases with decrease of centrality (cf. Fig. 2 (b)) the $\left(d n_{c h} /\left.d \eta\right|_{\eta=0}\right) /\left(0.5<N_{\text {part }}>\right)$ from JPCIAE with method c is close to the PHENIX data. If $d n_{c h} /\left.d \eta\right|_{\eta=0}$ from EKRT model was not normalized by $\left\langle N_{\text {part }}>\right.$ from method b, as did in [7], but by $<N_{\text {part }}>$ from method d the results of $\left(d n_{c h} /\left.d \eta\right|_{\eta=0}\right) /\left(0.5<N_{\text {part }}>\right)$ might have somewhat similar centrality dependence as in PHENIX data. Therefore one learns here that it is hard using $\left(d n_{c h} /\left.d \eta\right|_{\eta=0}\right) /\left(0.5<N_{\text {part }}>\right)$ as a function of $<N_{\text {part }}>$ to distinguish various theoretical models for particle production since $\left\langle N_{\text {part }}\right\rangle$ is not a well defined physical variable.

In summary, we used a hadron and string cascade model, JPCIAE, to investigate the energy and centrality dependences of charged particle pseudorapidity density at midpseudorapidity in relativistic $p+\bar{p}$ and $A+A$ collisions. Both the relativistic $p+\bar{p}$ experimental data and the PHOBOS and PHENIX data of $A u+A u$ collisions at RHIC could be reproduced fairly well within the framework of the JPCIAE model without retuning any parameter. The JPCIAE model predictions for full RHIC energy $A u+A u$ collisions and for $\mathrm{Pb}+\mathrm{Pb}$ collisions at the ALICE energy are also given. This study shows that since $<N_{\text {part }}>$ is not a well defined physical variable both experimentally and theoretically it may be hard to use charged particle pseudorapidity density per participant pair at mid-pseudorapidity as 
a function of $\left\langle N_{\text {part }}>\right.$ to distinguish various theoretical models for particle production.

Finally, the financial supports from NSFC in China and DOE in USA are acknowledged.

[1] B. B. Back, et al., PHOBOS collab., Phys. Rev. Lett. 85, 3100 (2000).

[2] K. Adcox, et al.,, PHENIX collab., Phys. Rev. Lett. 86, 3500 (2001).

[3] L. van Hove, Phys. Lett. B 118, 138 (1982).

[4] M. I. Gorenstein, Phys. Lett. B 281, (1992).

[5] J. I. Kapusta and A. P. Vischer, Phys. Rev. C 52, 2725 (1995).

[6] Xin-Nian Wang and M. Gyulassy, Phys. Rev. Lett. 86, 3496 (2001).

[7] K. J. Eskola, K. Kajantie and K. Tuominen, Phys. Lett. B 497, 39 (2001).

[8] D. Kharzeev and M. Nardi, nucl-th/0012025.

[9] A. Capella and D. Sousa, nucl-th/0106066.

[10] Ben-Hao Sa, An Tai, Hui Wang and Feng-He Liu, Phys. Rev. C 59, 2728 (1999);

Ben-Hao Sa and An Tai, Phys. Rev. C 62, 044905 (2000).

[11] B. B. Back, et al., PHOBOS collab., nucl-th/0105011.

[12] T. Sjöstrand, Comp. Phys. Comm. 82, 74 (1994).

[13] J. Cugnon, T. Mizutain, and J. Vandermeulen, Nucl. Phys. A 352, 505 (1981).

[14] G. F. Bertsch and S. Das Gupta, Phys. Rep. 160, 189 (1988);

A. Bonasera, F. Gulminelli, and J. Molitoris, Phys. Rep. 243, 1(1994).

[15] An Tai and Ben-Hao Sa, Comp. Phys. Comm. 116, 353 (1999).

[16] L. Ahle, et al., E802 Collab., Phys. Rev. C 59, 2173 (1999).

[17] Ben-Hao Sa, Yu-Ming Zheng, and Xiao-Ze Zhang, Phys. Rev. C 40, 2680 (1989).

[18] Hong Pi, Z. Phys. C 57, 485 (1993).

Key words: charged multiplicity; pseudorapidity density; number of participant nucleon; string; JPCIAE model. 